\title{
Q \\ RMA \\ Osteometría de falanges proximales de camélidos sudamericanos modernos. Variabilidad, estándares métricos y su importancia como conjunto comparativo para la interpretación de restos hallados en contextos arqueológicos
}

Dossier - Arqueología

\author{
Andrés D. Izeta', Clara Otaola ${ }^{2}$ y Alejandra Gasco ${ }^{3}$
}

\begin{abstract}
${ }^{1}$ CONICET, Museo de Antropología, Facultad de Filosofía y Humanidades, Universidad Nacional de Córdoba. E-mail: andresizeta@gmail.com

${ }^{2}$ CONICET, Museo de Historia Natural de San Rafael. E-mail: claraotaola@yahoo.com.ar ${ }^{3}$ CONICET, Instituto de Arqueología y Etnología, Facultad de Filosofía y Letras,

Universidad Nacional de Cuyo. E-mail: soljandra@yahoo.com.ar
\end{abstract}

\begin{abstract}
Resumen
Las medidas obtenidas a partir de huesos del esqueleto apendicular de camélidos sudamericanos han sido utilizadas en los últimos años como insumo básico para interpretar distintos aspectos relacionados con su asignación especifica, manejo de rebaños y estrategias económicas, entre otros. La forma y el tamaño de los huesos han sido interpretados como el resultado de distintas fuerzas asociadas a distintos tipos de restricciones ambientales o modificaciones producto del manejo humano. Esto es de gran importancia a la hora de interpretar la historia de la relación humano-camélido, dado que el registro material obtenido en depósitos arqueológicos posee variados restos a través de los cuales se pueden reconstruir diversas situaciones ocurridas en el pasado, tanto a nivel de paleo-economías o paleoambientes. Gran parte de estas interpretaciones se han basado en escasas medidas de estándares moderno. Por ello, este trabajo tiene como objetivo presentar datos métricos obtenidos de individuos contemporáneos de camélidos sudamericanos y que han sido considerados relevantes para su uso como material comparativo. Con esto se intenta evaluar desde una mirada crítica el uso de estándares locales y no locales para la asignación específica de muestras arqueológicas. Con el fin de evaluar la variabilidad en la forma y el tamaño de las primeras falanges se aplica el Análisis de Componente Principal y de Conglomerados (UPGMA). Para ello se toman en cuenta diferencias de tamaño, forma y procedencia geográfica con el fin de observar el peso aportado por cada variable en la conformación de esta muestra. Como resultado se concluye que el uso de ambas técnicas más la procedencia geográfica de las muestras modernas permite una definición por especie de grano más fino.
\end{abstract}

Palabras clave: camélidos sudamericanos, osteometría, esqueleto postcraneal, falange proximal, variabilidad, estándares comparativos.

South American camelids proximal phalanges osteometry. Variability, metric standards and its importance as a comparative set for the interpretation of remains from archaeological contexts.

\begin{abstract}
The measurements obtained from South American camelids appendicular skeleton bones have been used in recent years as a benchmark for interpreting different aspects of archaeological studies. The shape and size of bones have been linked to various factors which may be related to environmental restrictions and human management. This is of great importance for interpreting the history of human-camelid relationships through time, since the many remains obtained from archaeological deposits permit reconstructing situations that occurred in the past, which can be interpreted as the result of paleoeconomic activity or environmental changes. Therefore, this paper aims to present metric data obtained from contemporary individuals of South American camelids, which have been considered relevant for use as comparative material. An evaluation of this data is given a critical look in order to rethink the use of local and nonlocal standards for "taxonomic" assignment of archaeological samples. In order to assess the variability in shape and size of the first phalanx, the application of Principal Component Analysis and Cluster Analysis (UPGMA) is presented. This takes into account differences in size, shape and geographical origin in order to observe the contribution of each variable in the formation of this sample. As a result, it is concluded that the use of both techniques, as well as data from the geographical provenience of the modern samples, allows a finer-grained species determination.
\end{abstract}

Keywords: South American camelids, osteometry, postcranial skeleton, proximal phalanx, variability, comparative standards.

Recibido 20-08-2009. Recibido con correcciones16-11-2009. Aceptado 20-11-2009 
En los últimos años se ha extendido el uso de valores obtenidos a través de técnicas osteométricas. Tanto es así que en los últimos 15 años el uso de variables métricas han sido utilizadas para la definición de especies o morfotipos en más de 29 contextos diferentes del Noroeste argentino a los que hay que agregarle los casos del Perú, Bolivia y norte de Chile (Izeta 2008). Es por ello que el estudio de las variables métricas de huesos se vuelve una herramienta importante a los fines de poder alcanzar una definición de tamaños, y mediante ellos asociarlo a una especie determinada, mediante la cual pueda accederse a la resolución de diversos problemas arqueológicos.

La discriminación de camélidos por caracteres morfológicos y métricos ha sido desde hace ya unas décadas centro de discusión para arqueólogos y biólogos. En especial cuando se trata del análisis de conjuntos arqueológicos o paleontológicos ya que la sistemática de esta familia aun no ha sido resuelta. Sin embargo existen diversas preguntas que pueden ser respondidas sólo con la efectiva diferenciación de las especies de camélidos sudamericanos. Por ello se han efectuado distintas aproximaciones que han permitido en mayor o menor grado lograr exitosamente esta tarea. Varios caracteres morfológicos dentarios, craneanos y post-craneanos han sido definidos (e. g. Benavente et al. 1995, Wheeler 1982). No obstante muchos de ellos no contestan la amplia gama de variabilidad observada en poblaciones actuales. Otro tipo de aproximación utilizada es el análisis de fibras de camélidos el cual ha sido puesto a prueba en diversos conjuntos andinos y patagónicos ofreciendo en la actualidad una idea de la variabilidad de este tipo de restos y ayudando a diferenciar especies en una amplia gama de contextos (e.g. Reigadas 2001).

Por último existe una aproximación métrica basada en la toma de medidas de variables continuas en huesos, básicamente del esqueleto post-craneal. Estas técnicas eran ya conocidas en el ámbito de la paleontología argentina desde la década de 1980 en donde se utilizó con el fin de diferenciar un camélido extinguido de las pampas de la Argentina (Menegaz et al. 1989).

Asimismo estas técnicas permitieron comenzar a plantear la presencia de distintos grupos de tamaños dentro de los conjuntos arqueológicos a partir de los cuales aproximarnos a las respuestas que estábamos buscando como ¿cuándo comenzó el proceso de domesticación? ¿Cuál fue la estrategia predominante durante el paso de la caza y recolección a la producción? ¿Cuántos tipos de camélidos domésticos hubo en el pasado?

Desde una perspectiva histórica del desarrollo de las investigaciones arqueológicas en Sudamérica la diferenciación de camélidos ha sido y es un tema relevante. Esto puede observarse en la creciente utilización de este tipo de metodologías las cuales se encuentran formando parte de casi todos los protocolos de investigación, particularmente en contextos del Noroeste argentino, Norte de Chile, Bolivia y Perú, aunque su utilización ya está siendo aplicada en Patagonia, Pampa, las Sierras Centrales y Cuyo.

Ahora bien, para diferenciar a los camélidos del pasado es imperante saber cómo se comportan métricamente las poblaciones actuales razón por la cual se comenzó a recolectar este tipo de datos en muestras actuales de la Argentina y Perú. Por ello en la década de 1970 y 1980 se relevaron métricamente un gran número de camélidos de la Reserva de La Raya en la puna peruana (Miller 1979, Kent 1982). Estas muestras estaban integradas por ejemplares de llama, alpaca y vicuña locales. No se incluían guanacos debido a la ausencia de este animal en la zona en la actualidad. Por ello en la década de 1990 Mengoni Goñalons y Elkin tomaron medidas del esqueleto post-craneal de un guanaco (Lama guanicoe) de las Cumbres Calchaquíes (Pcia. de Salta) (Elkin 1996) con el fin de utilizarlas como estándar métrico local ya que se conocía la diferencia de tamaños entre esta forma y la de los guanacos patagónicos. Este set de datos fue el único disponible durante varios años hasta que se agregaron los datos obtenidos por Izeta (2004) de otro ejemplar de guanaco de la Falda Occidental del Aconquija (ubicada en la Provincia de Catamarca, 40/50 km al sur de donde se recolectó el otro ejemplar moderno). Si bien los ejemplares son pocos y con seguridad no representen la variabilidad esperada para una población, es lo más cercano que se tiene en la actualidad de los tamaños de camélidos que se pueden hallar en el área Andina. Esto último es de vital importancia ya que varios autores han tomado como estándar de animales silvestres a las medidas obtenidas en guanacos provenientes de Patagonia los que son de mayor tamaño que el más grande de los camélidos andinos como ya ha sido notado en varias oportunidades (e.g. Elkin et al. 1991, Izeta 2007, Mengoni 2008, Mengoni y Yacobaccio 2006).

En el año 1991 Elkin et al. popularizaron un modo de escalar el tamaño corporal de los camélidos del NOA sugiriendo un gradiente de tamaño que partía desde los camélidos más pequeños ( $V$. vicugna) hasta llegar a los más grandes (L. glama). Partiendo de ello y siguiendo una secuencia de tamaño corporal creciente los camélidos del área andina deberían dividirse en camélidos pequeños, incluyendo a vicuñas y alpacas ( $V$. pacos) y camélidos grandes que incluían al guanaco y a la llama, en ese orden.

Este gradiente de tamaño basado en los tamaños corporales de animales vivos actuales fue directamente utilizada como patrón para establecer estándares de variables métricas a nivel de elementos óseos basándose en datos obtenidos de un solo ejemplar de Lama guanicoe procedente del NOA (ver más arriba). Tanto es así que actualmente ese mismo individuo sigue siendo el estándar métrico mediante el cual se suele segregar entre 
especies de camélidos grandes (e.g. Grant 2008, Mengoni y Yacobaccio 2006). Sin duda ese ejemplar aun en la actualidad tiene que cargar con toda la variabilidad de la especie en sus hombros, razón por la cual es necesaria una agenda que permita comprender de un modo más cabal cuales son los tamaños de los huesos de esta especie en términos de población y no sólo de un individuo. Esta situación dista mucho de ser resuelta pero de a apoco se ha comenzado a tomar conciencia de esta falencia, lo que con seguridad traerá aparejado un aumento de la muestra y con ello sin duda también lo hará la variabilidad hallada al interior de la especie como ya se hizo notar en conjuntos de guanacos patagónicos (L'Heureux 2007).

El gradiente de tamaño de Elkin et al. (1991) difiere de los utilizados previamente por investigadores norteamericanos que habían trabajado con camélidos sudamericanos procedentes de contextos arqueológicos del Perú (e.g. Kent 1982, Miller 1979). Estos habían tomado como estándar comparativo a guanacos procedente de la Patagonia, ubicada miles de kilómetros al sur de su área de estudio. En términos de tamaño este guanaco era mayor al de las llamas actuales, razón por la que las clasificaciones y asignaciones de especie interpretadas a partir de análisis métricos asociaban a los elementos más grandes a la especie silvestre de los camélidos grandes. Sin duda este es un problema que ha sido remarcado en los últimos tiempos y una evidencia más de la necesidad de ampliar la base de datos regional de los tamaños de los huesos de camélidos sudamericanos y si estos permiten realizar asignaciones de tamaño y asociado a ello a asignaciones de especie.

Anteriormente hicimos notar este gradiente de tamaño basándonos en datos métricos de camélidos actuales de varias regiones de Sudamérica, apoyando mediante datos osteométricos al modelo de gradiente de tamaño (Cardich e Izeta 1999-2000).

Entonces, casi veinte años después del establecimiento del gradiente de tamaño ¿qué sabemos acerca de la variabilidad de estas especies? ¿Sigue siendo útil usar las variables métricas disponibles del único ejemplar de guanaco andino como un estándar confiable para segregar entre especies?

En este trabajo intentaremos responder a estas preguntas utilizando una base de datos ampliada de camélidos sudamericanos modernos tomando en cuenta la especie a la que pertenecen, la procedencia geográfica y el marco temporal de las muestras utilizadas.

\section{Materiales y métodos}

Los datos utilizados en este trabajo fueron obtenidos por diversos investigadores, por lo que no debe descartarse que algunas de las diferencias o similitudes que se observen a los largo de este trabajo pueda ser subsumida a algún tipo de error interobservador. De todos modos conociendo esta limitación y con el fin de tener una muestra un poco más representativa que un sólo ejemplar es que se utilizan datos de al menos 17 individuos.

La procedencia también es variada, tanto en términos de las regiones de donde procede, su adscripción temporal y los repositorios en donde se encuentran los ejemplares.

De la colección del Museo de Antropología (FFyH, UNC) se utilizan los datos de un guanaco andino y cuatro llamas. Se utilizan datos obtenidos de ejemplares alojados en la Sección Mastozoología del Museo Argentino de Ciencias Naturales "Bernardino Rivadavia" de la ciudad de Buenos Aires. De esta colección se utilizan los datos de una alpaca y dos llamas. Del Museo de La Plata (FCNyM, UNLP) se utilizan los datos de tres vicuñas peruanas y un guanaco patagónico, todos alojados en la División Paleontología de Vertebrados al momento de su consulta en 1999. También se utilizan datos de guanacos y vicuñas del Museo de Historia Natural de San Rafael (Mendoza), de la Facultad de Filosofía y Letras de la Universidad Nacional de Cuyo (Mendoza), de la Sección de Mastozoología de la Colección Boliviana de Fauna y del proyecto arqueológico Jach'a Marka, Tiwanaku, Bolivia (McGill University). También se utilizan datos recabados por Belotti (2007).

Los ejemplares proceden de zonas ecológicas tales como Puna Normal (Oruro, Bolivia), Puna Seca (Susques y Abra Pampa, Jujuy), Puna Salada (Laguna Blanca, Catamarca) y Valles y Quebradas (Quebrada de Humahuaca, Jujuy; Valle del Cajón, Catamarca). Además hay ejemplares del sur de San Juan y de la Patagonia. Estas zonas poseen distintos niveles sobre el nivel del mar y distintos climas, obteniéndose una representación de ejemplares que permiten trabajar con un gradiente latitudinal y otro altitudinal (Figura 1).

Los datos osteométricos fueron obtenidos siguiendo las variables propuestas por Kent (1982) (Figura 2). Estas son: 1: largo máximo, tomado en forma paralela al eje mayor utilizando como base la tangente formada por los cóndilos plantares proximales y el final en el punto más distal de la superficie articular distal (FP1V1 y BP1V177 de Kent [8]; Gl de von den Driesch [2])

2: ancho de la superficie articular proximal (FP1V2 y BP1V178 de Kent [8]; BFp de von den Driesch [2])

3: alto de la superficie articular proximal (FP1V3 y BP1V179 de Kent [8])

4: ancho de la superficie articular distal (FP1V4 y BP1V180 de Kent [8]; Bd de von den Driesch [2])

5: alto de la superficie articular distal (FP1V5 y BP1V181 de Kent [8])

En total se utilizan 485 observaciones (Tabla 1 y Tabla 2) sobre las cuales se realizaron análisis multivariados exploratorios y estadísticos con el fin de observar tendencias en los agrupamientos de los elementos. Se 


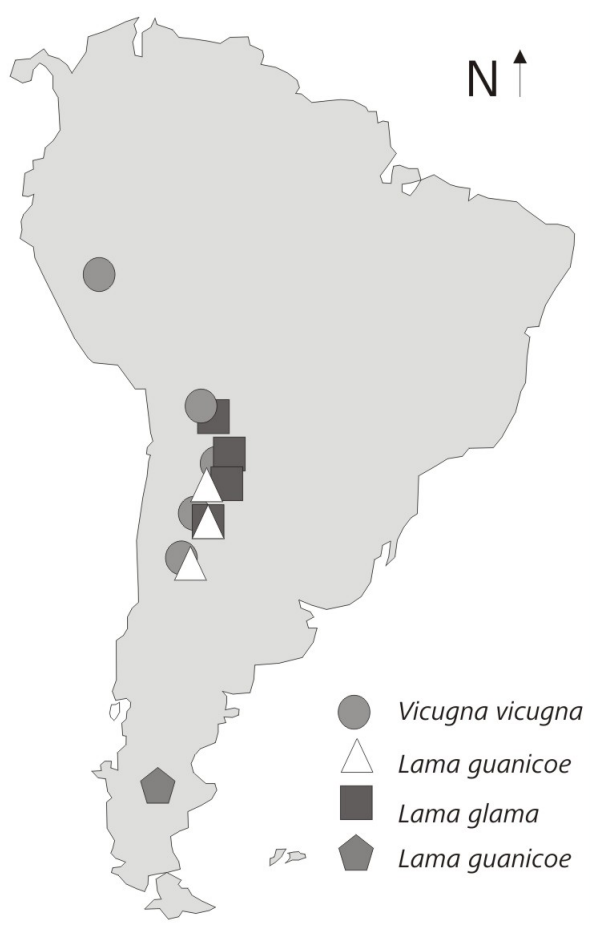

Figura 1. Procedencia de las muestras utilizadas en el trabajo.

utilizaron Análisis de Componente Principal (ACP) y de Conglomerados (UPGMA) (Izeta 2004, 2007, Menegaz et al. 1989), Medias Geométricas (Izeta 2009, L'Heureux 2005, 2007) y Mixture analysis (Yacobaccio 2008, 2009). Por otro lado se presenta una representación en un gráfico cartesiano de dos variables con el fin de observar la incidencia del tamaño en las clasificaciones específicas de los elementos.

Los análisis se realizaron utilizando el software PAST versión 1.94b (Hammer et al. 2001).

\section{Discusión}

En base a los datos relevados veremos cómo se comportan las muestras en dos dimensiones: el tamaño y la forma. Para el primer caso utilizamos un recurso gráfico de fácil visualización en el que se observa el gradiente de tamaño que parte de los camélidos más pequeños a los más grandes. En todos los casos tratamos los datos de las falanges delanteras separados de los datos obtenidos de las falanges traseras ya que estas pueden ser segregadas en base a su morfología (Kent 1982).

\section{Tamaño}

En primer término se presentan los gráficos bivariados en los que se tomaron como variables el largo máximo (\#1) y el ancho de la superficie articular proximal (\#2).

Para las falanges del apéndice anterior puede observarse una clara separación entre los elementos de los camélidos pequeños de los grandes (Figura 3). Dentro del grupo de los pequeños, se observa que los elementos de alpaca (VP) son más grandes que los de vicuña. Sin embargo debemos tener en cuenta el tamaño de la muestra ya que ha sido demostrado que los valores de vicuñas y alpacas pueden

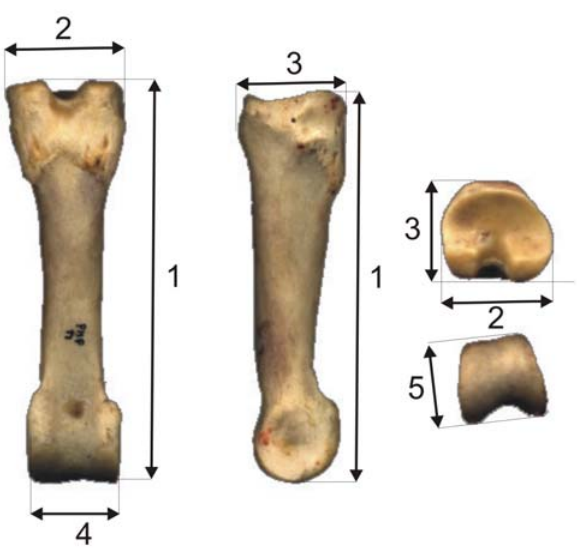

Figura 2. Variables utilizadas en este trabajo. Ver en el texto la definición de cada una de ellas.

superponerse (Cartajena 2009). Al interior del grupo conformado por las vicuñas observamos una disposición de los valores que podría asociarse a variaciones subespecíficas o regionales. Recordemos que se han definido por caracteres morfológicos y genéticos dos subespecies de vicuña: V.V.vicugna Molina y V.v.mensalis. La primera habita en el sur de Bolivia, norte de Chile y noroeste argentino y la segunda habita en Perú (Vila 2006a). En estudios morfométricos se estableció que V.v.vicugna tiene un tamaño corporal mayor (Yacobaccio 2006), entonces se puede postular que a mayor latitud el tamaño corporal de los individuos aumenta y que ese aumento de tamaño corporal se verá reflejado en el esqueleto. Entonces podemos observar que las vicuñas de San Juan (VSJ) se ubican dentro de los elementos de mayor tamaño, en tanto las de latitudes bajas son las que presentan un tamaño menor (VH). Si bien las muestras son escasas nos permiten observar una primera tendencia dentro del grupo de los camélidos pequeños y que es lo que deberíamos esperar en cuanto a variaciones geográficas de esta especie. Por otro lado los elementos de alpaca (VP) son los más grandes dentro de este conjunto, con lo que se confirma esta gradiente de tamaño para los camélidos pequeños.

En el caso de los camélidos grandes no es tan fácil observar una diferencia entre grupos. Si siguiéramos la secuencia de gradientes de tamaño deberíamos tener elementos de guanaco norandino, llamas y luego guanacos patagónicos. Pero como puede observarse en la Figura 3 , los elementos se encuentran agrupados siguiendo otro criterio. Sin duda el problema que se presenta en este caso es la superposición con elementos de camélidos domésticos lo cual complejiza la situación, especialmente a la hora de realizar una asignación específica siguiendo el criterio de tamaño. De todos modos puede observarse una coherencia entre elementos correspondientes a animales cuya procedencia es de las mismas áreas geográficas y a pesar de la superposición entre los elementos de distintas especies resulta interesante observar que los camélidos silvestres respetan la secuencia de gradiente de tamaño según su correspondencia a área geográfica, siendo que 


\begin{tabular}{|c|c|c|c|c|c|c|c|c|c|c|}
\hline Taxón & Procedencia & Código & & 1 & 2 & 3 & 4 & 5 & MG & Datos \\
\hline ALPACA & BS AS & 48.354 .1 & VP & 65,04 & 18,24 & 16,66 & 15,65 & 15,03 & 1,719 & Izeta \\
\hline ALPACA & BS AS & 48.354 .2 & VP & 66,04 & 18,86 & 16,72 & 16,25 & 15,05 & 1,720 & Izeta \\
\hline ALPACA & BS AS & 48.354 .4 & VP & 64,95 & 17,91 & 16,68 & 16,20 & 15,16 & 1,722 & Izeta \\
\hline ALPACA & BS AS & 48.354 .5 & VP & 66,01 & 18,08 & 16,81 & 15,43 & 14,99 & 1,719 & Izeta \\
\hline GUANACO & PATAGONIA & MLP-G1 & GP & 75,00 & 22,00 & 21,00 & 19,00 & 17,00 & 1,762 & Izeta \\
\hline GUANACO & PATAGONIA & MLP-G2 & GP & 75,00 & 22,00 & 20,00 & 19,00 & 17,00 & 1,762 & Izeta \\
\hline GUANACO & PATAGONIA & MLP-G3 & GP & 73,00 & 23,00 & 19,00 & 19,00 & 17,00 & 1,762 & Izeta \\
\hline GUANACO & PATAGONIA & MLP-G4 & GP & 85,00 & 23,00 & 22,00 & 20,00 & 18,00 & 1,783 & Izeta \\
\hline GUANACO & PATAGONIA & MLP-G5 & GP & 74,00 & 24,00 & 19,00 & 19,00 & 17,00 & 1,762 & Izeta \\
\hline GUANACO & PATAGONIA & MLP-G6 & GP & 75,00 & 22,00 & 21,00 & 19,00 & 15,00 & 1,719 & Izeta \\
\hline LLAMA & $?$ & 33.61 .1 & LL & 75,84 & 20,65 & 20,95 & 17,70 & 18,01 & 1,783 & Izeta \\
\hline LLAMA & $?$ & 33.61 .2 & LL & 74,49 & 20,43 & 20,87 & 18,46 & 18,69 & 1,796 & Izeta \\
\hline LLAMA & $?$ & 33.61 .3 & LL & 74,41 & 21,29 & 20,55 & 18,13 & 17,23 & 1,767 & Izeta \\
\hline LLAMA & $?$ & 33.62 .1 & LL & 69,37 & 19,34 & 18,60 & 16,66 & 16,11 & 1,743 & Izeta \\
\hline LLAMA & $?$ & 33.62 .2 & LL & 69,67 & 19,87 & 18,13 & 16,76 & 16,11 & 1,743 & Izeta \\
\hline LLAMA & $?$ & 33.62 .3 & LL & 69,98 & 19,95 & 18,56 & 16,88 & 15,95 & 1,740 & Izeta \\
\hline LLAMA & $?$ & 33.62 .4 & LL & 69,40 & 19,10 & 18,47 & 16,05 & 15,98 & 1,741 & Izeta \\
\hline GUANACO & CATAMARCA & G149-4 & GC & 72,80 & 21,50 & 18,10 & 17,80 & 16,70 & 1,756 & Izeta 2004 \\
\hline GUANACO & CATAMARCA & G149-5 & GC & 71,90 & 21,50 & 18,40 & 18,70 & 16,30 & 1,748 & Izeta 2004 \\
\hline GUANACO & CATAMARCA & G149-6 & GC & 71,70 & 22,20 & 18,50 & 18,70 & 16,70 & 1,756 & Izeta 2004 \\
\hline GUANACO & CATAMARCA & G149-7 & GC & 72,80 & 21,80 & 18,40 & 18,20 & 16,90 & 1,760 & Izeta 2004 \\
\hline GUANACO & SALTA & G1100-1 & GS & 71,32 & 19,65 & 18,37 & 17,58 & 16,29 & 1,747 & Elkin 1996 \\
\hline GUANACO & SAN JUAN & FPDG-1 & GSJ & 78,36 & 22,65 & 21,44 & 19,07 & 17,72 & 1,777 & Otaola \\
\hline GUANACO & SAN JUAN & FPDG-2 & GSJ & 75,90 & 22,04 & 21,02 & 18,64 & 18,38 & 1,790 & Otaola \\
\hline GUANACO & SAN JUAN & FPDG-3 & GSJ & 77,72 & 22,67 & 20,53 & 19,20 & 18,37 & 1,790 & Otaola \\
\hline LLAMA & CATAMARCA & L148-3 & LLC & 75,40 & 21,80 & 19,40 & 17,90 & 18,00 & 1,783 & Izeta 2004 \\
\hline LLAMA & CATAMARCA & L148-4 & LLC & 76,30 & 21,90 & 18,30 & 17,70 & 15,70 & 1,735 & Izeta 2004 \\
\hline LLAMA & CATAMARCA & L151-1 & LLC & 78,65 & 24,08 & 22,68 & 17,39 & 16,15 & 1,744 & Srur e Izeta 2008 \\
\hline LLAMA & CATAMARCA & L151-2 & LLC & 79,31 & 22,05 & 20,91 & 17,64 & 15,74 & 1,735 & Srur e Izeta 2008 \\
\hline LLAMA & JESÚS DE MACHACA & $\| 1$ & LLO & 68,60 & 20,80 & 18,10 & 18,60 & 15,70 & 1,735 & Gasco \\
\hline LLAMA & JESÚS DE MACHACA & 112 & LLO & 69,10 & 21,20 & 19,60 & 18,70 & 15,60 & 1,732 & Gasco \\
\hline LLAMA & JESÚS DE MACHACA & 113 & LLO & 69,30 & 20,70 & 19,10 & 18,50 & 16,70 & 1,756 & Gasco \\
\hline LLAMA & JESÚS DE MACHACA & $\| 4$ & LLO & 68,30 & 21,80 & 19,30 & 18,50 & 16,10 & 1,743 & Gasco \\
\hline VICUÑA & HUANUCO & V999-10 & VH & 56,30 & 16,90 & 14,50 & 13,40 & 12,80 & 1,665 & Izeta \\
\hline VICUÑA & HUANUCO & V999-11 & VH & 60,70 & 17,50 & 16,30 & 14,60 & 13,70 & 1,688 & Izeta \\
\hline VICUÑA & HUANUCO & V999-12 & VH & 61,00 & 17,60 & 16,10 & 15,10 & 15,10 & 1,721 & Izeta \\
\hline VICUÑA & HUANUCO & V999-14 & $\mathrm{VH}$ & 62,20 & 17,80 & 16,30 & 14,10 & 13,20 & 1,675 & Izeta \\
\hline VICUÑA & HUANUCO & V999-5 & VH & 53,80 & 15,30 & 14,20 & 13,60 & 10,60 & 1,603 & Izeta \\
\hline VICUÑA & HUANUCO & V999-6 & VH & 54,90 & 15,40 & 13,90 & 13,50 & 10,60 & 1,603 & Izeta \\
\hline VICUÑA & HUANUCO & V999-7 & VH & 56,10 & 17,40 & 14,10 & 13,10 & 13,00 & 1,670 & Izeta \\
\hline VICUÑA & HUANUCO & V999-8 & VH & 55,50 & 14,30 & 15,10 & 14,00 & 13,00 & 1,670 & Izeta \\
\hline VICUÑA & HUANUCO & V999-9 & VH & 61,70 & 17,90 & 16,10 & 14,10 & 13,10 & 1,673 & Izeta \\
\hline VICUÑA & JUJUY & V500 & VJ & 53,50 & 16,70 & 15,00 & 14,40 & 13,20 & 1,675 & Elkin 1996 \\
\hline VICUÑA & ORURO & va1 & VO & 62,15 & 16,75 & 17,62 & 13,92 & 12,60 & 1,660 & Gasco \\
\hline VICUÑA & ORURO & va2 & Vo & 62,31 & 16,74 & 17,52 & 13,92 & 12,36 & 1,653 & Gasco \\
\hline VICUÑA & ORURO & va3 & VO & 61,90 & 17,12 & 17,55 & 14,17 & 12,57 & 1,659 & Gasco \\
\hline VICUÑA & ORURO & va4 & VO & 62,92 & 16,74 & 17,18 & 13,80 & 12,21 & 1,649 & Gasco \\
\hline VICUÑA & SAN JUAN & v1 & VSJ & 62,60 & 18,55 & 16,95 & 15,70 & 13,95 & 1,694 & Otaola \\
\hline VICUÑA & SAN JUAN & v2 & VSJ & 61,70 & 17,95 & 15,55 & 14,85 & 13,75 & 1,689 & Otaola \\
\hline VICUÑA & SAN JUAN & v6 & VSJ & 61,30 & 18,00 & 16,70 & 14,40 & 13,80 & 1,690 & Otaola \\
\hline
\end{tabular}

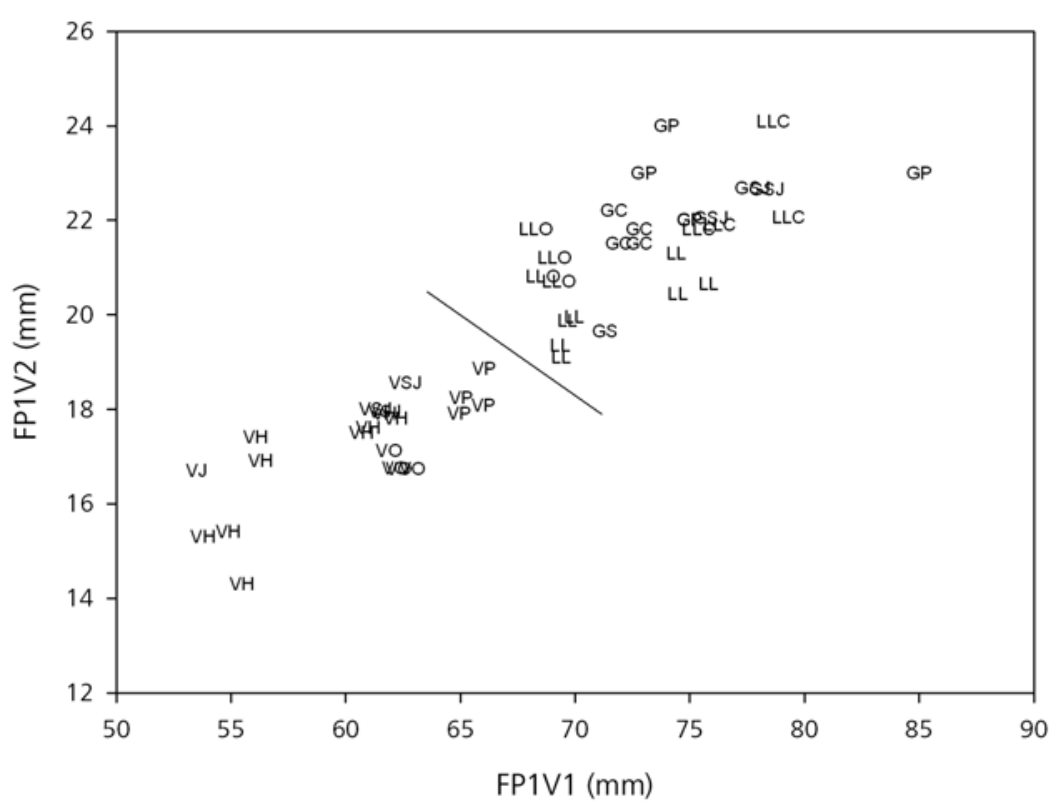

Tabla 1. Valores métricos y Medias Geométricas de falanges proximales delanteras utilizados en el presente trabajo.
GP Lama guanicoe (Patagonia)
GSJ Lama guanicoe (San Juan)
GS Lama guanicoe (Salta)
GC Lama guanicoe (Catamarca)
LL Lama glama (?)
LLC Lama glama (Catamarca)
LLO Lama glama (Jesús de Machaca)
vP Vicugna pacos
VH Vicugna vicugna (Huánuco)
vo Vicugna vicugna (Oruro)
vJ Vicugna vicugna (Jujuy)
vsJ Vicugna vicugna (San Juan)

Figura 3. Gráfico bivariado (apéndices delanteros). 


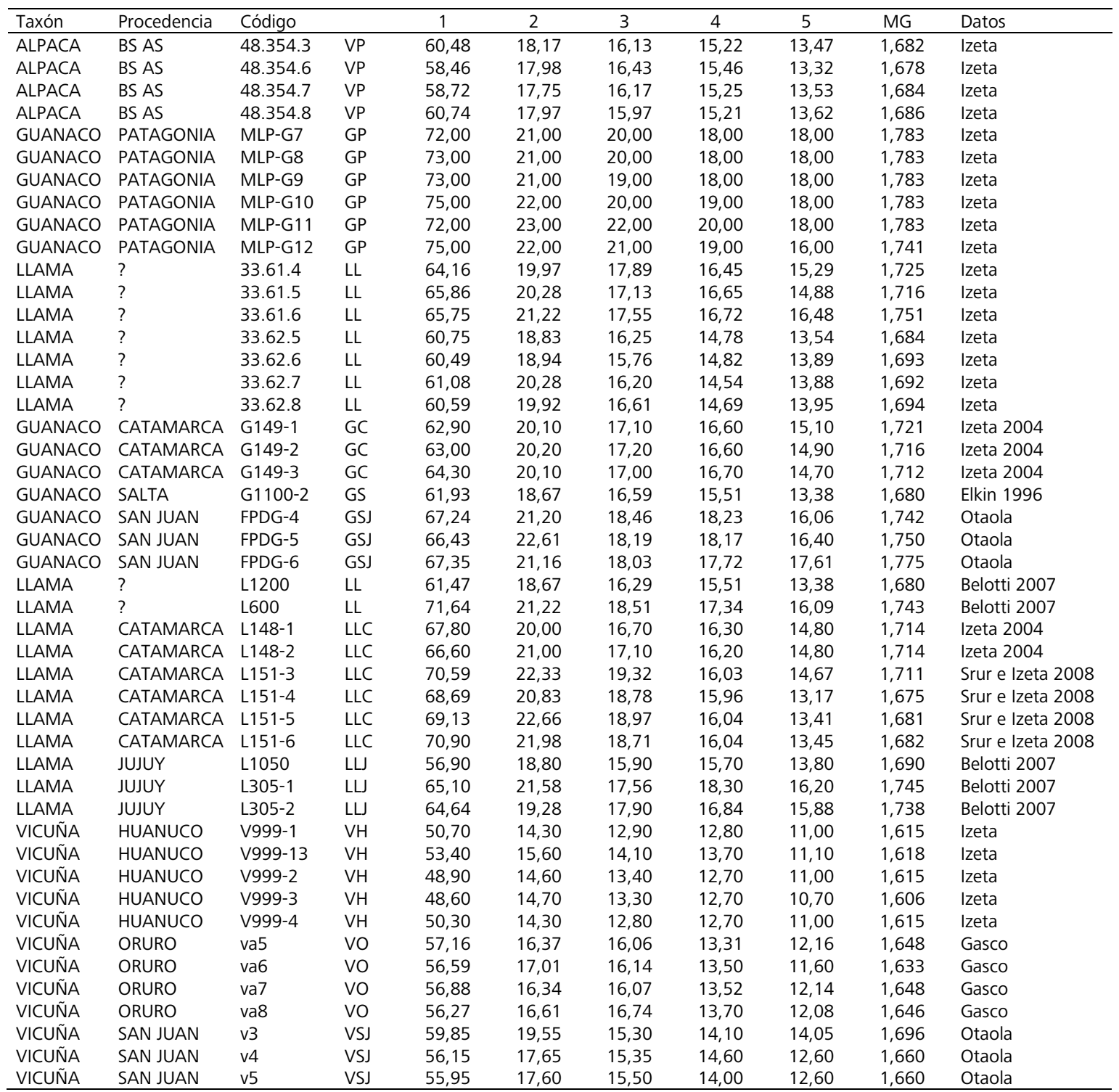

Tabla 2. Valores métricos y Medias Geométricas de falanges proximales traseras utilizados en el presente trabajo.

los guanacos norandinos (GC, GS) son los más pequeños, le siguen los guanacos de San Juan (GSJ) y por último los patagónicos (GP). Esto mismo puede verse en los ejemplares domésticos entre las llamas de Oruro y las de Catamarca.

Mediante esta aproximación no se puede observar una clara separación entre llamas y guanacos. Sin embargo si puede observarse que las llamas y guanacos de más al sur son de tamaño más grande que las del norte. Una diferenciación clara y que puede tener importancia para el sur de los Valles Calchaquíes (Provincias de Catamarca y Tucumán, Noroeste de la Argentina) es la que se produce entre los elementos de llama y guanacos del área. Aquí podemos comenzar a visualizar la importancia de contar con colecciones de referencia locales y que veremos a los largo de este trabajo.

El resto de los elementos nos muestra una gran variabilidad de los tamaños en las dos especies por lo cual a la hora de utilizar estos sets de datos debemos tener en cuenta la procedencia de los animales que se van a comparar.

Para las falanges de los apéndices posteriores (Figura 4) Podemos observar las primeras "anomalías" dentro de este esquema de camélidos pequeños y grandes. Un elemento de llama adulta procedente de Jujuy se ubica en un sector intermedio entre vicuñas y alpaca. Es interesante integrar este dato ya que aporta datos métricos a la variabilidad observada de los camélidos domésticos históricos y modernos (Yacobaccio 2009). Por otro lado un elemento de un ejemplar de vicuña de San Juan se ubica junto con elementos de llama y de guanacos andinos. Nuevamente los valores de guanaco andino vuelven a superponerse con otros que corresponden a llamas. Como en los resultados de los apéndices anteriores los guancos andinos y las llamas de los valles Calchaquíes no vuelven a superponerse (GC y LLC).

Entonces de modo preliminar puede observarse que en términos generales esta primera aproximación permite una segregación confiable entre camélidos pequeños y 


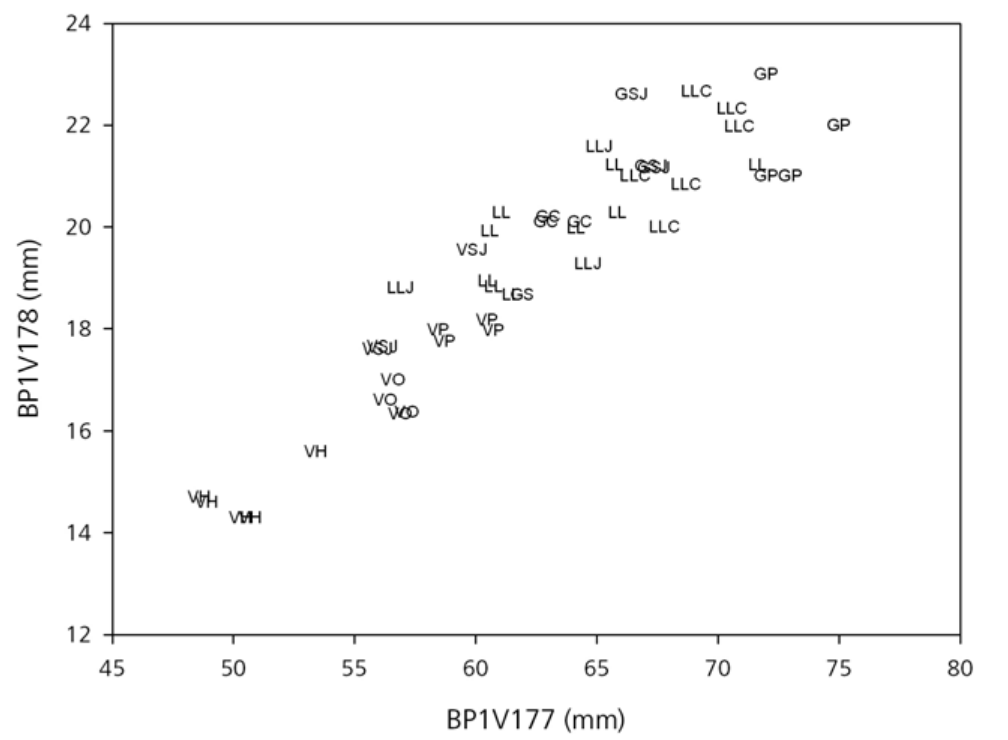

GP Lama guanicoe (Patagonia)
GSJ Lama guanicoe (San Juan)
GS Lama guanicoe (Salta)
GC Lama guanicoe (Catamarca)
LL Lama glama (?)
LLJ Lama glama (Jujuy)
LLC Lama glama (Catamarca)
VP Vicugna pacos (?)
VH Vicugna vicugna (Huánuco)
Vo Vicugna vicugna (Oruro)
VSJ Vicugna vicugna (San Juan)

Figura 4. Gráfico bivariado (apéndices traseros). grandes para elementos delanteros. Por otro lado, para los elementos traseros esta situación se torna un poco más compleja al tener una mayor variabilidad en los tamaños de algunas especies (Ilama y vicuña)

Avanzando con el uso de las variables veremos los resultados del Análisis de Conglomerados (UPGMA). Para este caso se utilizaron las cinco variables descriptas más arriba.

Para los elementos de los apéndices anteriores podemos observar que los camélidos pequeños y los camélidos grandes se separan en dos grandes bloques (Figura 5). Como se había observado en el gráfico bivariado el corte entre ambos grupos es neto y ninguno de los ejemplares de camélidos pequeños es agrupado con los de camélidos grandes y viceversa. Es difícil hacer una interpretación de los agrupamientos en este caso ya que no sabemos cual es el peso de las variables en este agrupamiento. En el caso de los gráficos bivariados pudimos esbozar una interpretación biogeográfica muy simple a partir de los valores métricos y su ubicación dentro de un gradiente de tamaño corporal creciente.

No obstante lo anterior es interesante observar que tomando una mayor cantidad de variables la asignación a los grupos (pequeño y grande) es correcta en todos los casos, aunque estos agrupamientos aun no permiten asignar con mayor confianza una muestra incógnita (como la sería una muestra arqueológica) alguno de los subgrupos que se forman al interior de las dos grandes categorías.

De todos modos y en este caso en particular creemos que lo que se puede observar mediante el uso de esta técnica es que las "membresías" o asignaciones a grupos a veces son forzadas por lo cual su poder exploratorio es limitado. Si bien en el caso de los camélidos pequeños no parece existir problemas de asignaciones, en el grupo de los más grandes el panorama no es auspicioso.
En el caso de los elementos de los apéndices traseros la segregación no es tan clara (Figura 6). Esto ya lo habíamos notado en un trabajo anterior (Srur e Izeta 2008) en el cual los elementos de los apéndices anteriores parecen discriminar mejor entre los conjuntos conformados por las distintas especies. En concreto existen elementos de vicuña asociados con llamas, alpacas junto con llamas y casi todas las posibilidades de combinación posibles. De todos modos cabe aclarar que existe una combinación de datos que está integrando ejemplares de distintas procedencias latitudinales y altitudinales, lo que probablemente estén afectando los modos en que se agrupan los elementos.

Otro de los análisis utilizados para explorar el agrupamiento de los ejemplares según las variables métricas utilizados es el uso de Análisis de Componentes Principales.

Nuevamente comenzamos con los elementos del esqueleto apendicular delantero pudiendo observar que el primer componente es quien explica más del 96\% de la varianza de la muestra (Tabla 3). Esto nos permite interpretar que el tamaño (que es el que generalmente representa este primer componente) es quien tiene más peso en el agrupamiento de los elementos.

Teniendo en cuenta lo anterior vemos nuevamente la segregación entre camélidos pequeños y grandes, ubicándose las vicuñas y alpaca en los cuadrantes de la izquierda del gráfico y los camélidos grandes a la derecha (Figura 7). Este análisis de tipo exploratorio vuelve a confirmar que el tamaño es una de las variables de mayor peso en la conformación de grupos y de asociaciones de elementos.

Para los elementos de los miembros traseros se observa nuevamente la presencia del elemento de llama de Jujuy entre los camélidos pequeños y la asociación de algunas llamas de tamaño relativamente pequeño junto 

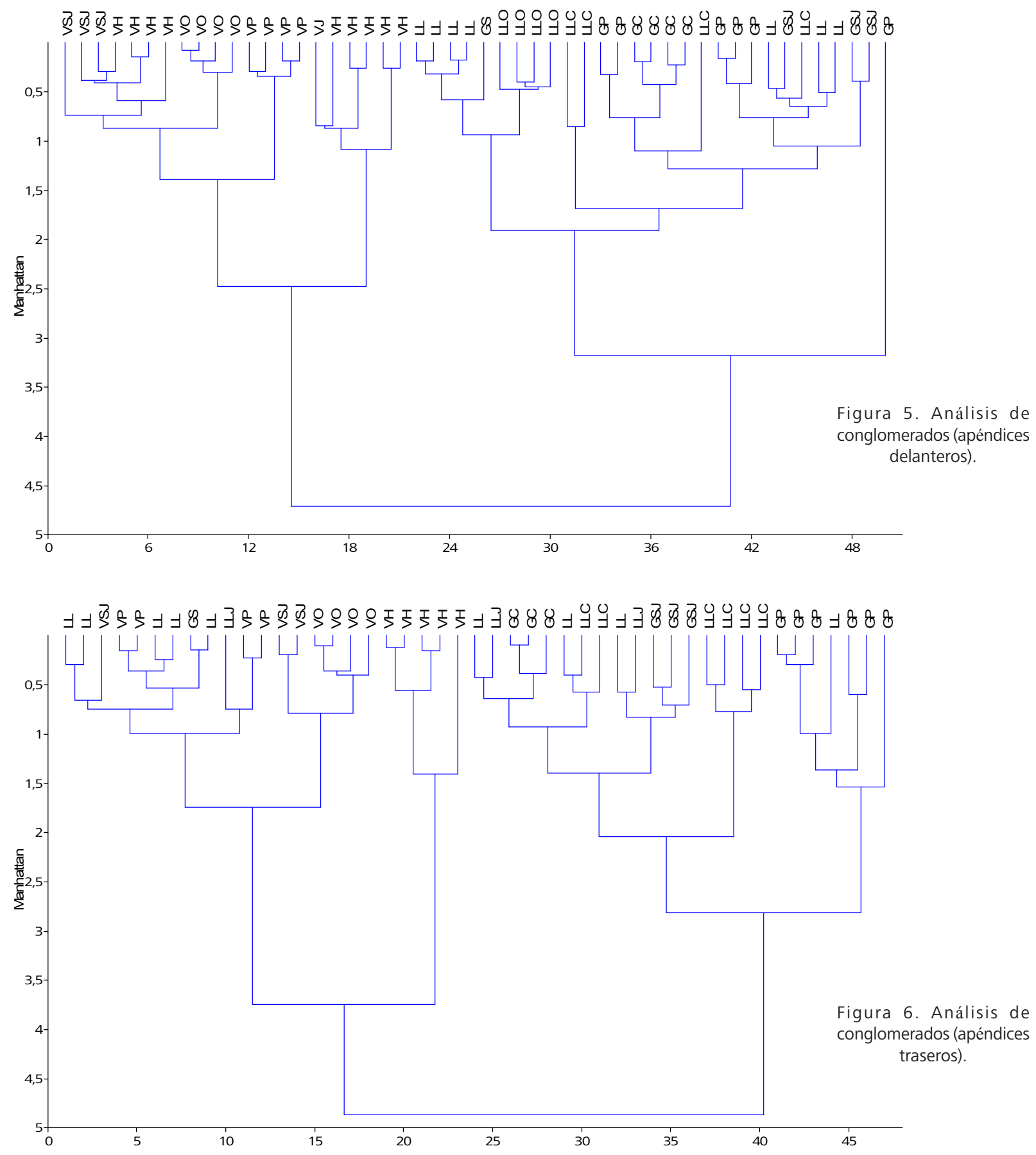

con los elementos de alpaca (Figura 8). Esto puede contrastarse con los gráficos bivariados, observándose un comportamiento similar ya que las diferencias se basan en el tamaño de los elementos óseos tal como puede observarse en los autovalores que arroja el ACP de estos elementos (Tabla 4). Nuevamente vemos que las falanges traseras no son buenas discriminadoras entre especies.

\section{Forma}

Teniendo en cuenta la importancia del tamaño para la segregación de elementos y conociendo que el tamaño es afectado por diversos parámetros ambientales es que observaremos el comportamiento de la forma.
Con el fin de evaluar la forma tratando de eliminar el efecto del tamaño se utiliza el valor calculado de la Media Geométrica (MG) para cada uno de ellos (Izeta 2009, L'Heureux 2005, 2007). Con los valores obtenidos se crearon dos gráficos de cajas mediante los cuales se trata de establecer la variabilidad de la forma en cada una de las especies. Asimismo con esa matriz de datos se procedió a realizar un Mixture Analysis con el fin de observar los agrupamientos que se producen en cuanto a las formas de los elementos.

Como se puede observar en el resultado de las MG para los elementos delanteros y traseros (Figura 9 y 10) la segregación no es concluyente en muchos de los casos. 


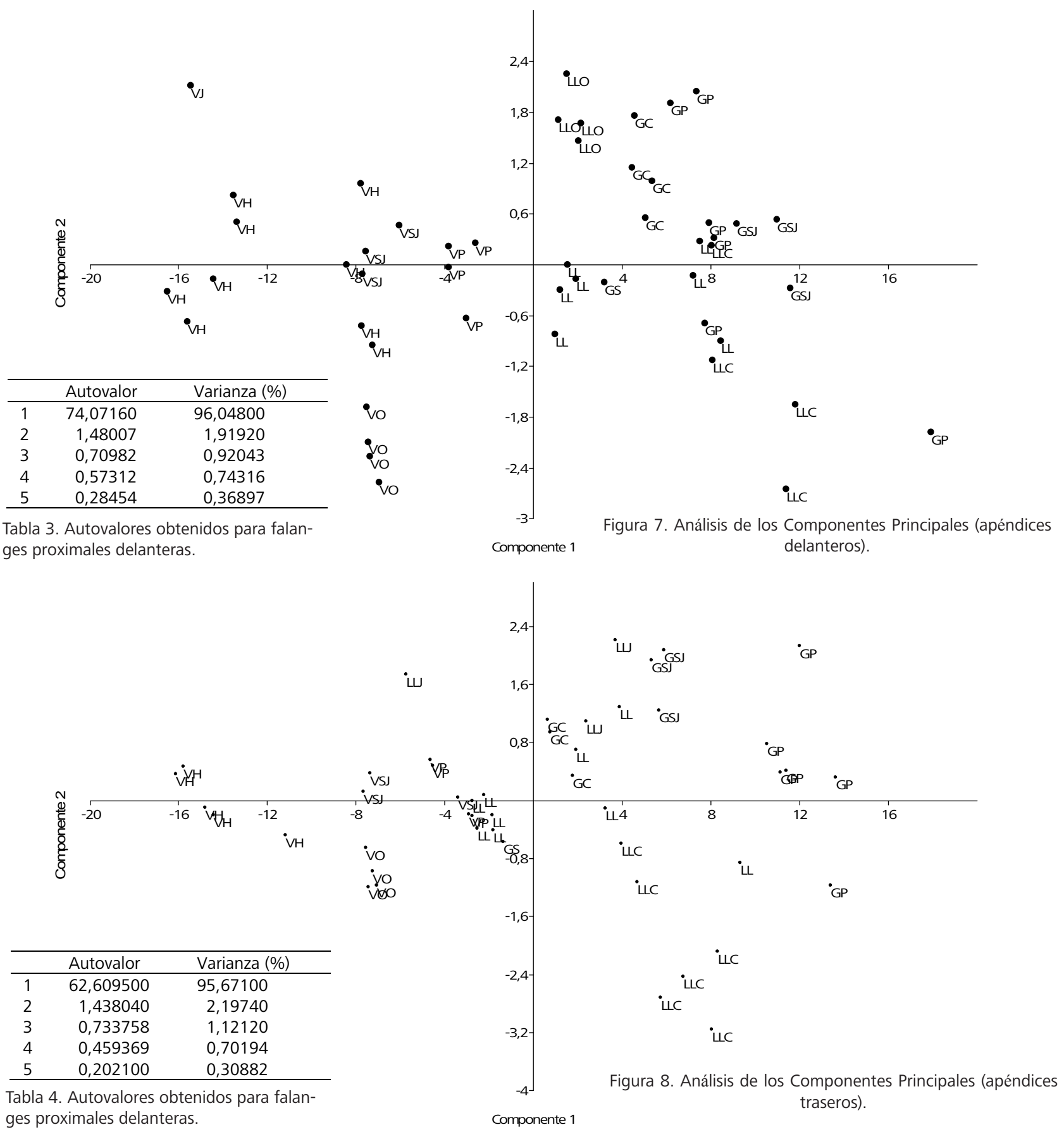

Para los camélidos pequeños tenemos que las vicuñas de Huánuco $(\mathrm{VH})$ poseen una variabilidad tal que es igual a la que presentan para los elementos delanteros las vicuñas de otras regiones geográficas e incluso la alpaca. No obstante ello, el resto de los ejemplares se segrega según su región geográfica de procedencia. Con ello podemos observar que la forma (que esta genéticamente controlada) permite separar entre especies de un modo mas confiable.

Para los elementos delanteros de los camélidos grandes observamos que los ejemplares tienen valores de MG similares ofreciendo un panorama bastante homogéneo.

En el caso de los elementos traseros se puede observar que para los guanacos se presenta una segregación de las formas según su área de procedencia. Las Ilamas presentan un panorama más homogéneo siendo sus valores de MG mas similares entre si.

Utilizamos el Mixture Analysis (Yacobaccio 2008, Monchot 2008) con el fin de establecer si la forma permitiría una asignación específica de elementos. Para ello tomamos la matriz de datos construida con los valores calculados de MG para cada elemento.

Esto nos permitió observar que los valores de MG pueden dividirse en tres grupos (definidos a priori) y que responden a distribuciones normales de los valores. En la Tabla 5 puede observarse que los elementos delanteros de 


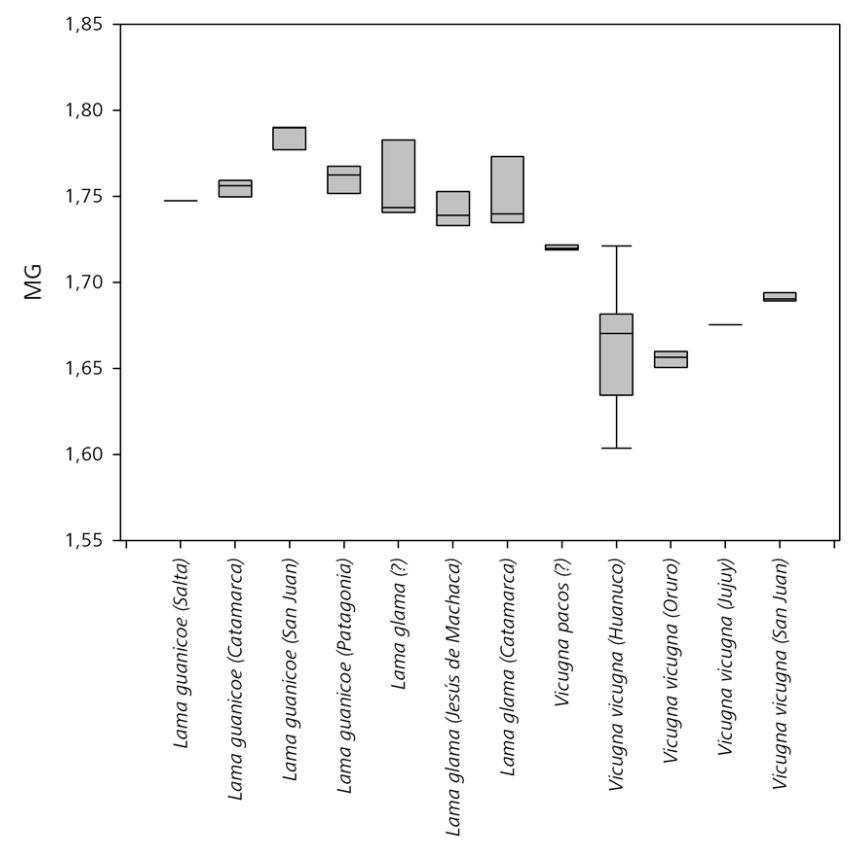

Figura 9. Media Geométrica (apéndices delanteros)

guanacos forman un grupo, las llamas un segundo y las vicuñas y alpacas un tercero. Para los elementos traseros el panorama vuelve a ser poco claro en cuanto a las asignaciones (Tabla 6), aunque existen agrupaciones entre guanacos, llamas y vicuñas, siendo los más complejos de interpretar los casos de la llama de Jujuy (L1050) y el guanaco de Salta (G1100-2).

\section{Tamaño, forma y algo más...}

Los resultados muestran una variabilidad amplia en el conjunto de camélidos en general. En casi todos los casos se pudo visualizar una separación clara entre los camélidos pequeños y los grandes. No obstante ello se ha registrado casos de elementos de especie conocida que se agruparon con el grupo de camélidos opuesto al que deberían haberse asignado. Ese es el caso de la falange trasera L1050 procedente de Jujuy. Esto no deja de alertarnos sobre la gran variabilidad que tiene esta familia tanto en tamaños como en formas de los elementos.

\begin{tabular}{lrrr}
\hline & \multicolumn{1}{c}{ Grupo 1 } & \multicolumn{1}{c}{ Grupo 2 } & \multicolumn{1}{c}{ Grupo 3 } \\
\hline LGS & 0,000000075 & 5,571000000 & 7,218000000 \\
LGC & 0,000000001 & 0,000387000 & 7,392000000 \\
LGSJ & 0,000000000 & 0,000000000 & 2,214000000 \\
LGP & 0,000000000 & 0,000000002 & 6,909000000 \\
LL & 0,000000504 & 26,400000000 & 6,834000000 \\
LLC & 0,000004217 & 19,240000000 & 6,184000000 \\
LLO & 0,000002710 & 25,060000000 & 6,338000000 \\
VP & 0,006481000 & 0,000000000 & 2,694000000 \\
VH & 10,550000000 & 0,000000000 & 0,014850000 \\
VO & 4,252000000 & 0,000000000 & 0,001528000 \\
VJ & 10,570000000 & 0,000000000 & 0,031180000 \\
VSJ & 3,842000000 & 0,000000000 & 0,209000000 \\
\hline
\end{tabular}

Tabla 5. Mixture analysis. Asignaciones a grupos de valores de MG de falanges proximales delanteras.

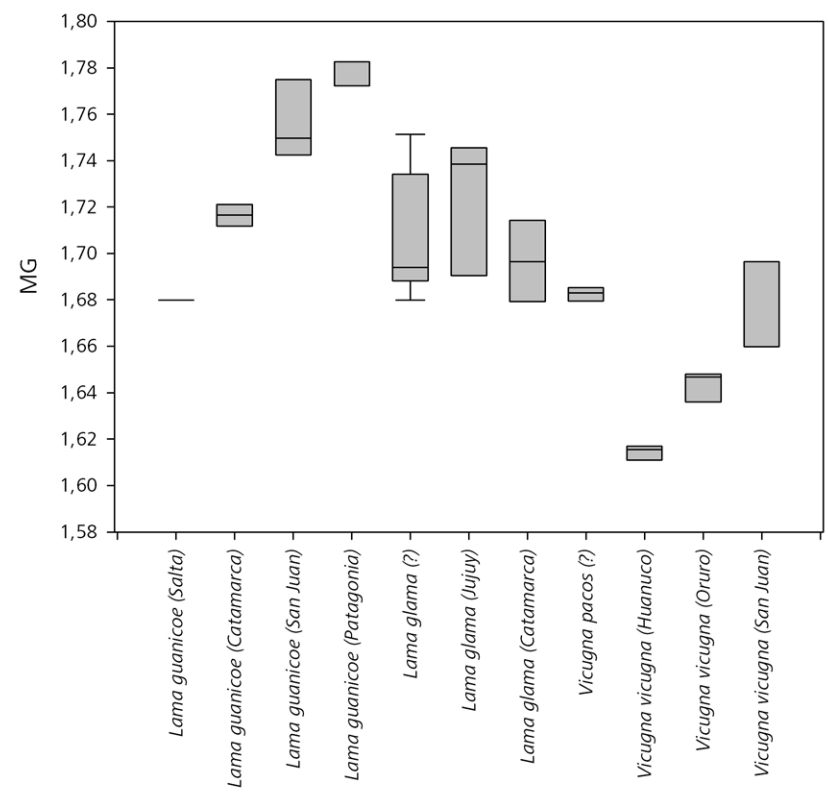

Figura 10. Media Geométrica (apéndices traseros)

De todos modos es interesante observar como se agrupan los elementos del miembro apendicular delantero de vicuña independientemente de su procedencia y su asignación a distintas subespecies (Vila 2006b). En este caso tenemos un gradiente latitudinal amplio (desde los $10^{\circ}$ lat S hasta los $32^{\circ}$ lat S) que parece no afectar demasiado a la definición del grupo de camélidos de tamaño más pequeño. Por el otro lado el caso de las falanges primeras posteriores parece no ser tan coherente como se observa en los resultados obtenidos en el elemento anterior. Lo mismo sucede tanto con guanacos como con llamas.

En cuanto a los camélidos de tamaño grande (llamas y guanacos) el agrupamiento para la falange primera anterior se presenta más compleja que para el caso de las vicuñas debido a que existe superposición entre valores. De todos modos esto puede explicarse a través de variaciones regionales y etáreas de las muestras. En este caso las Ilamas de mayor tamaño (códigos 148 y 151 procedentes de Catamarca -Puna y valles-) se agrupan

\begin{tabular}{lrrr}
\hline & Grupo 1 & \multicolumn{1}{c}{ Grupo 2 } & \multicolumn{1}{c}{ Grupo 3 } \\
\hline LGS & 1,423000000 & 0,367100000 & 8,677000000 \\
LGC & 0,022310000 & 3,499000000 & 0,006099000 \\
LGSJ & 0,000044010 & 5,560000000 & 0,000000000 \\
LGP & 0,000000000 & 1,984000000 & 0,000000000 \\
LL & 0,400800000 & 1,086000000 & 13,060000000 \\
LLJ & 0,000469100 & 5,631000000 & 0,000000000 \\
LLC & 0,309500000 & 1,273000000 & 9,369000000 \\
VP & 1,125000000 & 0,473300000 & 13,260000000 \\
VH & 2,249000000 & 0,000078780 & 0,000000000 \\
VO & 5,395000000 & 0,009732000 & 0,000000416 \\
VSJ & 4,204000000 & 0,048950000 & 0,004690000 \\
\hline
\end{tabular}

Tabla 6. Mixture analysis. Asignaciones a grupos de valores de MG de falanges proximales traseras. 
con muestras de guanaco de mas al sur (San Juan) lo que puede resultar lógico si asumimos que a mayor latitud (y climas mas fríos) se produce un incremento en el tamaño de los individuos (regla de Bergmann 1847). Misma situación sucede con llamas de latitudes mas bajas (procedentes de Oruro, código "va") y llamas juveniles (código 150) respecto de guanacos de latitudes mas altas (Catamarca, Salta y Tucumán, códigos g1100, g149). Como se dijo anteriormente, esta situación no es tan evidente en las falanges del miembro apendicular trasero aunque pueden interpretarse algunos agrupamientos como resultado de los mismos factores que se observaron más arriba, especialmente si tomamos en cuanta la forma de los elementos.

Como conclusión preliminar de este análisis puede estimarse que la falange delantera discriminaría de un modo más efectivo a los elementos según su asignación por especie utilizando los análisis que se presentaron en este trabajo. Esto último es de gran utilidad a la hora de comparar con elementos de los cuales no se tiene una asignación específica concreta como es el caso de los restos hallados en contextos arqueológicos.

Por otro lado observamos que si se tiene en cuenta la procedencia geográfica de los ejemplares comparativos aun puede mantenerse la idea del gradiente de tamaño para algunas áreas en particular como los valles Calchaquíes. Evidentemente los guanacos de San Juan son de mayor tamaño que los de Salta/Catamarca por lo que sería ideal utilizar estos ejemplares como estándares para los restos hallados en esa provincia y para el norte de Mendoza. Esto no hace más que confirmar la necesidad de una mayor cantidad de ejemplares comparativos para más áreas.

Creemos, entonces, que la utilización de material comparativo moderno es una herramienta importante a la hora de evaluar composiciones taxonómicas de conjuntos no actuales, aunque se debe proceder con cautela ya que como se mostró en este trabajo existen diferencias entre muestras comparativas actuales de distintas regiones.

\section{Agradecimientos}

Este proyecto ha sido financiado por CONICET y ANPCyT-FONCYT PICT 31633. A todos aquellos que nos permitieron el acceso a muestras comparativas o datos inéditos: Andrés Laguens, Mirta Bonnin, Mariana Dantas y Carlos Belotti López de Medina. A José Hierling, Gabriela Srur y Luis Viaña por su colaboración en la obtención y procesamiento de muestras. David Flores (MACN), Susana Bargo (Museo de La Plata, FCNyM, UNLP) por el acceso al relevamiento de ejemplares alojados en sus instituciones. Asimismo agradecemos a Julieta Vargas encargada de la Sección de Mastozoología de la Colección Boliviana de Fauna, en donde se encuentra depositado el ejemplar de vicuña y a Claudine Vallières (McGill University), del proyecto arqueológico Jach'a Marka, Tiwanaku, Bolivia, quien gentilmente nos permitió medir su ejemplar de llama.

\section{Bibliografía}

Benavente A., M. A., L. Adaro A., P. Gecele C., y C. Cunazza P. 1993. Contribución a la determinación de especies animales en arqueología: familia camelidae y taruca del norte. Universidad de Chile, Vicerrectoría académica y estudiantil, Departamento Técnico de Investigación, Santiago.

Bergmann, C. 1847: Über die Verhaltnisse der Warmeokonomie der Thiere zu ihrer Grosse. Göttingen Studien 1: 595-708.

Cardich, A. y A. D. Izeta. 1999-2000. Revisitando Huargo (Perú). Análisis cuantitativos aplicados a restos de Camelidae del Pleistoceno Tardío. Anales de Arqueología y Etnología 54-55: 29-40. U N Cuyo. Mendoza.

Cartajena, I. 2009. Explorando la variabilidad morfométrica del conjunto de camélidos pequeños durante el Arcaico Tardío y el Formativo Temprano en Quebrada Tulán, norte de Chile. Revista del Museo de Antropología 2:199-214.

Elkin, D. C. 1996. Arqueozoología de Quebrada Seca 3: indicadores de subsistencia humana temprana en la Puna Meridional Argentina. Tesis para otra al grado de Doctor de la UBA. F. F. y L. Buenos Aires, Argentina. MS

Elkin, D. C., C. M. Madero, G. L. Mengoni Goñalons, D. E. Olivera y H. D. Yacobaccio. 1991. Avances en el estudio arqueológico de los camélidos en el noroeste argentino. Actas de la VII Convención Internacional de Especialistas en Camélidos Sudamericanos.

Grant, J. L. 2008 El recurso Camelidae en sitios de la Puna Meridional Argentina: Una aproximación osteométrica. Tesis de Licenciatura, FFyL, Universidad de Buenos Aires.

Hammer, Ø., Harper, D.A.T., and P. D. Ryan, 2001. PAST: Paleontological Statistics Software Package for Education and Data Analysis. Palaeontologia Electronica 4(1): 9pp. http://palaeo-electronica.org/2001_1/past/issue1_01.htm

Izeta, A. D. 2004. Zooarqueología del Sur de los Valles Calchaquíes: Estudio de conjuntos faunísticos del Periodo Formativo. Tesis doctoral inédita, UNLP.

Izeta, A. D. 2007. Zooarqueología del sur de los valles Calchaquíes (Provincias de Catamarca y Tucumán, República Argentina): Análisis de conjuntos faunísticos del primer milenio A.D. B.A.R. Int. Series S1612, Oxford.

Izeta, A. D. 2008. Variabilidad osteométrica de camélidos de sitios arqueológicos del NOA. I Congreso Nacional de Zooarqueología Argentina. Malargue, Mendoza. 
Izeta, A. D. 2009. Variabilidad osteométrica de camélidos de sitios arqueológicos del NOA. Zooarqueología a principios del siglo XXI: aportes teóricos, metodológicos y casos de estudio, M. De Nigris, P. M. Fernández, M. Giardina, A. F. Gil, M. A. Gutiérrez, A. Izeta, G. Neme y H. D. Yacobaccio (Ed).

Kent, J. D. 1982. The Domestication and exploitation of the South American camelids: methods of analysis and their application to circum-lacustrine archaeological sites in Bolivia and Peru. Tesis de Doctorado, Washington University, St. Louis, Missouri, USA. Ms.

L'Heureux, G. L. 2005. Variación morfométrica en restos óseos de guanaco de sitios arqueológicos de Patagonia austral continental y de la Isla Grande de Tierra del Fuego. Magallania 33 (1): 81-94.

L'Heureux, G. L. 2007. La reducción del tamaño de los guanacos (Lama guanicoe) entre el Pleistoceno Final y el Holoceno en el extremo austral de Patagonia continental. Archaeofauna 16 (2007): 173-183.

Menegaz, A., F. J. Goin y E. Ortiz Jaureguizar. 1989 Análisis morfológico y morfométrico multivariado de los representantes fósiles y vivientes del género Lama (Artiodactyla, Camelidae). Sus implicancias sistemáticas, biogeográficas, ecológicas y biocronólogicas. Ameghiniana 26(3-4): 153-172.

Mengoni Goñalons, G. L. 2008. Camelids in ancient Andean societies: A review of the zooarchaeological evidence. Quaternary International 185: 59-68.

Mengoni Goñalons, G. L. y H. D. Yacobaccio. 2006. The domestication of South American camelids. A view from the South-Central Andes. En: Zeder, M., D. Bradley, E. Emshwiller y B. Smith (Eds), Documenting domestication. New genetic and archaeological paradigms. UCP.

Miller, G. 1979. An introduction to the ethnoarchaeology of andean camelids. PhD Dissertation. University of California, Berkeley.

Monchot, H. 2008. Les chasseurs tardigravettiens de la Baume de Goulon (Salernes, Var, France). Revue de
Paléobiologie, 27 (2): 409-427.

Reigadas, M. C. 2001. Variabilidad y cambio cultural en el NOA desde los comienzos de la domesticación animal hasta la consolidación de las adaptaciones pastoriles. Tesis para optar al grado de Doctor de la UBA. F. F. y L. UBA. Buenos Aires. MS.

Srur, M. G. y A. D. Izeta. 2008. Osteometría de elementos del esqueleto apendicular de Lama glama. Su importancia como conjunto comparativo para estudios arqueológicos. Resúmenes XXII Jornadas Argentinas de Mastozoología. Villa Giardino, Córdoba. Pp. 116-117.

Vilá, B. (Ed.). 2006a. Investigación, conservación y manejo de vicuña. Proyecto MACS-Argentina-INCO-Unión Europea. Buenos Aires, 208 pp.

Vilá, B. 2006b. Suborden Tylopoda. En: Mamíferos de Argentina. Sistemática y distribución. R.M. Bárquez, M.M. Díaz y R. A. Ojeda (Ed.). SAREM. Pp. 117-118.

Von den Driesch, A. 1976 A Guide to the Measurement of Animal Bones from Archaeological Sites. Bulletin No. 1, Peabody Museum of Archaeology and Ethnology. Cambridge, USA.

Wheeler, J. 1982. Ageing Llamas and Alpacas by their teeth. Llama World 1: 12-17.

Yacobaccio, H. D. 2006. Variables morfométricas de vicuñas (Vicugna vicugna vicugna) en Cieneguillas, Jujuy. En: Vilá, B. (Ed.). Investigación, conservación y manejo de vicuñas. Proyecto MACS-Argentina-INCO-Unión Europea. Buenos Aires. Pp 101-112.

Yacobaccio, H. D. 2008. Morfometría de llamas (Lama glama) y sus aplicaciones arqueológicas. Resúmenes del I Congreso Nacional de Zooarqueología Argentina. Pp43.

Yacobaccio, H. D. 2009. Osteometría de llamas (Lama glama L.) y sus consecuencias arqueológicas. Zooarqueología a principios del siglo XXI: aportes teóricos, metodológicos y casos de estudio, M. De Nigris, P. M. Fernández, M. Giardina, A. F. Gil, M. A. Gutiérrez, A. Izeta, G. Neme y H. D. Yacobaccio (Ed). 